\section{Downregulation of SRPK2 promotes cell cycle arrest through E2F1 in non-small cell lung cancer}

\author{
Xin Li, ${ }^{1}$ Shaoyu Yang, ${ }^{1}$ Minna Zhang, \\ Shuhuan Xie, ${ }^{3}$ Zefeng Xie ${ }^{3 *}$ \\ ${ }^{1}$ Department of Oncology, Affiliated \\ Hangzhou First People's Hospital, \\ Zhejiang University School of Medicine, \\ Hangzhou \\ ${ }^{2}$ Department of Oncology, Hangzhou \\ Cancer Hospital, Hangzhou \\ ${ }^{3}$ Department of Thoracic Surgery, First \\ Affiliated Hospital of Medical College of \\ Shantou University, Shantou, China
}

\section{Abstract}

Serine-arginine protein kinase (SRPK) belongs to a class of cell cycle regulating kinases that can phosphorylate proteins containing serine/arginine-Rich (SR) regions. SR proteins are a family of RNAbinding phosphoproteins that control both constitutive and alternative pre-mRNA splicing events. However, little is known about their role in non-small cell lung cancer (NSCLC). In the present study, we found that serine-arginine protein kinase 2 (SRPK2) expression was upregulated in NSCLC tissues compared with adjacent normal tissues. Kaplan-Meier curve analyses showed that the overall survival time of NSCLC patients with high SRPK2 expression was shorter than those with low SRPK2 expression. Overexpression of SRPK2 promoted NSCLC cell proliferation and cell cycle arrest, while knockdown of SRPK2 inhibited proliferation and promoted cell cycle arrest in NSCLC cell lines. SRPK2 promoted the transcriptional regulation of $E 2 F 1$ on downstream cell cycle related genes through phosphorylation of SC35. Xenograft model showed that SRPK2 promoted tumor growth in vivo. SRPK2 phosphorylated SC35 and phosphorylated SC35 activated E2F1 transcription of cyclin-related proteins, thereby promoting the cycle progression of NSCLC. Our findings demonstrated that SRPK2 may be a potential therapeutic target for NSCLC clinical therapy, which plays an important role in the progression of NSCLC.

\section{Introduction}

Lung cancer is currently one the main leading cause of cancer-related death, which characterized by rapid growth, migration, invasion and recurrence. ${ }^{1}$ Nonsmall cell lung cancer (NSCLC) is the most common malignant tumor in lung cancer and the leading cause of cancer-related death worldwide, accounting for $80-85 \%$ of all lung cancers. ${ }^{2}$ However, the clinical outcome of conventional therapies remains very poor. This is largely due to the fact that most patients are diagnosed with the disease either at the locally advanced or metastatic stages. $^{3}$ Despite significant advances in molecular marker diagnosis and related targeted therapies in recent years, there is still much improvement in the treatment of NSCLC. ${ }^{4,5}$ However, the survival rate is still low, with a 5 -year survival rate of about $15 \%{ }^{6}$

Increased serine-arginine protein kinase (SRPK) pre-mRNA splicing regulators have been reported to contribute to cancer development and metastasis. ${ }^{7}$ Ser/Arg-Rich (SR) proteins are an important class of nonsnRNP splicing factors that play an important role in constitutive splicing and alternative splicing and mRNA export. ${ }^{8}$ The SRPK belongs to a class of cell cycle-regulating kinases that phosphorylate proteins containing the SR region. ${ }^{9}$ SRPK phosphorylates the SF2/ASF and regulates its nuclear translocation. ${ }^{10}$ Serine-arginine protein kinase 2 (SRPK2) is a member of SRPK, and is reported to be involved in cell cycle and apoptosis. ${ }^{11}$ SRPK2 binds to acinus proteins and phosphorylates acinus, thereby promoting up-regulation of cyclin A1 and promoting leukemic cell proliferation, while knocking down SRPK2 or acinus, results in $G_{1}$ arrest in cells. ${ }^{12}$ In neurons, SRPK2 T492 is phosphorylated by AKT and then translocated into the nucleus to phosphorylate SC35. Phosphorylated SC35 inhibits p53 activation, thereby promoting the up-regulation of CyclinD1 expression in the nucleus, promoting the proliferation of nerve cells, and ultimately leading to the apoptosis of nerve cells related to Alzheimer's disease. ${ }^{13}$ SRPK2 promotes tumor cell growth and metastasis in colorectal cancer, In addition, SRPK2 is also involved in proliferation, migration, invasion, cycle and apoptosis of prostate cancer cells. ${ }^{14,15}$ The above studies indicate that SRPK2 may play a role in regulating the biological behavior of cancer cells and promoting the development of various tumors. Studies have found that SRPK2 is upregulated in $94 \%$ NSCLC and $68 \%$ of lung squa-
Correspondence: Zefeng Xie, Department of Thoracic Surgery, First Affiliated Hospital of Medical College of Shantou University, 57 Changping Road, Jinping District, Shantou City, Guangdong Province, China.

Tel. +86.0754.88905178.

E-mail: ZefengXieshj@163.com

Key words: SRPK2; NSCLC; E2F1; cell cycle.

Contributions: XL, ZX, experiments concept and design; SX, experiments performing; SY, $M Z$, analysis and interpretation of experiments results. All the authors have read and approved the final version of the manuscript and agreed to be accountable for all aspects of the work.

Conflict of interest: The authors declare that they have no competing interests, and all authors confirm accuracy.

Availability of data and materials: All data generated or analyzed during this study are included in this published article.

Informed consent: Written informed consent was obtained from all patients participating in this study, which was approved by the Ethics Committee of First Affiliated Hospital of Shantou University Medical College.

Received for publication: 29 August 2019. Accepted for publication: 25 November 2019

This work is licensed under a Creative Commons Attribution-NonCommercial 4.0 International License (CC BY-NC 4.0).

CCopyright: the Author(s), 2019

Licensee PAGEPress, Italy

European Journal of Histochemistry 2019; 63:3067 doi:10.4081/ejh.2019.3067

mous cell carcinoma. ${ }^{16}$ The upregulation of SRPK1 mRNA and protein promotes the growth and migration of cancer cells and may play a carcinogenic role in NSCLC. ${ }^{17}$ However, the function of SRPK2 in NSCLC has not been reported. The E2F transcription factor was originally identified as an activator of adenoviral transcription and belongs to the E2F protein family. ${ }^{18}$ E2F1-3 interact with the Rb protein family to inhibit the transcriptional activity of E2Fs in early $G_{0}$ or $G_{1}$. E2Fs are released when the cell cycle progresses to the $G_{1}$ phase and $\mathrm{Rb}$ is phosphorylated by Cdks. ${ }^{19}$ Studies have shown that the transcription factor E2F1 is up-regulated in some types of tumors, while expression levels are very low in lung adenocarcinoma and squamous cell carcinoma as well as in low-grade 
neuroendocrine tumors (typical and atypical carcinoid). ${ }^{20}$ Consistent with the proliferation role of E2F1 in Large cell neuroendocrine carcinoma (LCNEC) and small cell lung cancer (SCLC), it was also found that the E2F1 protein status is directly related to the expression of some transcriptional targets (such as cyclin $\mathrm{E}$ and $\mathrm{p} 45^{\text {SKP2 }}$ ) involved in $\mathrm{S}$ phase progression. ${ }^{21} \mathrm{SRSF} 2$ has been found to be a novel target for $E 2 F 1$ in a variety of human lung cancer cell lines, including neuroendocrine lung cancer, and these two proteins have been shown to synergistically induce lung adenocarcinoma cells apoptosis. ${ }^{22}$ Therefore, this evidence suggests that $E 2 F 1$ plays an important role in cell cycle progression and apoptosis. Previous studies have shown that SC35 can interact with E2F1 to regulate the transcription function of $E 2 F 1$, while AKT inhibitors can reduce phosphorylation of SC35 and transcription of Cyclin E and p45 $5^{\text {SKP2 }}$ downstream of $E 2 F 1$, indicating that phosphorylated SC35 can activate E2F1 to affect downstream cyclins transcription, thereby promoting cell cycle progression..$^{23}$

In current study, our results showed that SRPK2 participates in the cell cycle progression and cell proliferation of NSCLC and explored the mechanism of SRPK2 regulating cell cycle related genes. SRPK2 phosphorylates SC35 and phosphorylated SC35 activates the transcriptional function of $E 2 F 1$ on cycle-associated proteins. Therefore, SRPK2 may play a key role in the progression of NSCLC and may be a potential therapeutic target for clinical treatment of NSCLC.

\section{Materials and Methods}

\section{Tissue samples gather and cell line culture}

The 60 paired samples of adjacent tissues of carcinoma and NSCLC were obtained from patients during operation. All patients in the study had no adjuvant therapy before surgery. Written informed consent was obtained from all patients participating in this study, which was approved by the Ethics Committee of First Affiliated Hospital of Shantou University Medical College. All tissue specimens were stored at $-80^{\circ} \mathrm{C}$ until use.

One human lung epithelial cell (BEAS2B) and five NSCLC cell lines (A549, SPCA1, SKMES1, CALU3, NCIH520 and NCHI1573), and HEK-293T were purchased from American Type Culture Collection (Manassas, VA, USA) and cultured in DMEM medium (Gibco, Gaithersburg, MD, USA; Cat. No: 670087) and 1640 medium (Gibco; Cat. No: 21870-
076) supplemented with $10 \%$ fetal bovine serum (FBS) (Gibco; Cat. No: 16140071) and added the $100 \mathrm{U} / \mathrm{mL}$ penicillin and 100 $\mathrm{Ug} / \mathrm{mL}$ streptomycin. The cells were cultured in a $5 \% \mathrm{CO}_{2}$ incubator at $37^{\circ} \mathrm{C}$.

\section{Cell treatments, plasmids and trans- fection}

The following plasmids were used for transient transfection, including pcDNA3.1, pcDNA3.1-SRPK2, pcDNA3.1SRPK2 ${ }^{\mathrm{T} 492 \mathrm{~A}}$, pcDNA3.1-SC35, pCMVE2F1 and pGL2-Luc, pGL2-cyclin E encodes a luciferase protein under the control of the Cyclin E promoter, the luciferase promoter region under the control of pGL2Skp2 human Skp2 encoding spans from 272 to +244 residues and pCMV-DP1.The derivable E2F-reactive structure encoding a firefly luciferase reporter gene was ligated to the tandem repeat of a specific E2F transcriptional response element (TRE) under the control of a basal promoter element (TATA box), purchased from SuperArray (Tebu-bio, Le Perray en Yvelines, France).

The specifically two target sequences of human SRPK2 RNA were purchased from Genechem (Shanghai, China). The specific sequences were as follows: 5'-UUAACAUUUAAAGACAAACCU-3' and 5'-GUUUGUCUUUAAAUGUUAAAG-3'. The negative control (NC) was 5'-TTCTCCGAACGTGTCACGT-3' respectively. Cells were transfected with siRNA oligonucleotide duplexes using lipofectamine 2000 (Invitrogen, Carlsbad, CA, USA) according to the manufacturer's instructions. Cells were transfected with siRNA oligonucleotides using oligofectamine reagent (Invitrogen) according to the manufacturer's instructions and subjected to cell analysis experiments $72 \mathrm{~h}$ after transfection.

\section{Cell proliferation and cell cycle analysis}

Cell proliferation assays was detected with 5-Bromo-2-deoxy-Uridine (BrdU, 11669915001, Roche, Basel, Switzerland). The cells were seeded into 24-well plates at $10^{4} /$ well and the cell density was 50\%-60\%. The plasmid was transfected; $24 \mathrm{~h}$ later, 10 $\mu \mathrm{m}$ of BrdU was added to each well and incubated for $4 \mathrm{~h}$. The cells were fixed with $4 \%$ cold paraformaldehyde for $30 \mathrm{~min}$ and then washed with PBS was for three times, $0.2 \%$ Triton X-100 was used for permeabilization for $10 \mathrm{~min}$. After cells were washed three times with PBS, BrdU antibody was diluted 1:1000 and $300 \mu \mathrm{L}$ per well was added into the cells and incubated at $4{ }^{\circ} \mathrm{C}$ overnight. After the cells were washed three times with PBS, cells were stained with DAPI for nuclear. Images were acquired by fluorescence microscope.
The collected cells were washed with cold PBS, and then fixed by $70 \%$ ethanol at $4^{\circ} \mathrm{C}$ overnight. The cells were incubated in the dark for $30 \mathrm{~min}$ at room temperature using staining buffer containing of $1 \mu \mathrm{g} / \mathrm{mL}$ PI and $10 \mathrm{mg} / \mathrm{mL}$ RNaseA (Sigma-Aldrich, St. Louis, MA, USA). Flow cell counting method Modifit LT3.1 (BD-Biosciences, Franklin Lakes, NJ, USA) equipped with CXP software was used to determine the percentage of cells in different phases of the cell cycle.

\section{qRT-PCR}

qRT-PCR was performed on an ABI 7500 system (Applied Biosystems, Carlsbad, CA, USA) using TransStart Tip Green qPCR SuperMix (Takara Bio Inc., Shiga, Japan) according to the manufacturer's instructions. Specific primers for mRNA amplification were as follows: GAPDH-forward: 5'-CGA-GAT-CCCTCC-AAA-ATC-AA-3'; GAPDH-reverse: 5'-ATC-CAC-AGT-CT-CTG-GGT-GG-3'; SRPK2-forward: 5'-CCG-CTT-CCG-CCGCGT-CGG-A-3'; SRPK2-reverse:5'CATCCAAGCTTTCTAAT-3'; CyclinEforward: 5'-GAA-ATG-GCC-AAA-ATCGAC-AG-3'; CyclinE-reverse:5'-TCTT T G - T C A - G G T-GT G - GGG - A - 3 '; p45 ${ }^{\text {SKP2 }}$ forward:5'-GCT-GAA-GAG-CAAAGG-GAG-TG-3'; p45 ${ }^{\text {SKP2 }}$ reverse:5'GAA-GGG-AGT-CCC-ATG-AAA-CA-3'. In all experiments, the reference gene GAPDH was used to quantify the level of each sample using qRT-PCR. By using the method that the ratio of target gene copy number to GAPDH mRNA copy number was multiplied by 100 to calculate the relative gene expression for each sample. $\Delta \mathrm{Ct}$ method was used for analysis of the data.

\section{Western blotting}

Western blotting was performed on total proteins from patient tissues, cultured cells and xenograft tumors tissues. The protein was separated by dodecyl sulfate, sodium salt-polyacrylamide gel electrophoresis (SDS-PAGE), then the protein expressions were detected using standard techniques for immunoassays. Antibodies to CyclinE (SAB, \#29030, 1:1000 dilution), SRPK2 (Sigma-Aldrich, \#HPA015522, 1:1000 dilution), p45 SKP2 (Invitrogen, \#323300, 1:2000 dilution), SC35 (Abcam, Cambridge, UK, \#ab204916, 1:2000 dilution), E2F1 (Abcam, \#ab179445, 1:2000 dilution). HRP-conjugated secondary antibodies (Invitrogen, G-21040) were used for the blotting. Controls were referred to $\beta$-actin (Cell Signaling Technology, Danvers, MA, USA; \#4970, 1:5000 dilution). The expression of each protein was analyzed using biometric digital image software and 
recorded as integral density (ID). The final result of the ratio of target proteins were calculated as ID (per protein) / ID ( $\beta$-actin) protein expression.

\section{Dual luciferase reporter assay}

After the HEK-293T cells (293T cells have high transfection efficiency and large base, which eliminates the individual differences caused by transfection efficiency.) were transfected for $48 \mathrm{~h}$ according to the group, $300 \mu \mathrm{L}$ of cell lysis buffer (Passive lysis buffer from Promega, Madison, WI, USA) was added to lyse the cells. The cells were centrifugated at 13,200 rpm for $2 \mathrm{~min}$ to remove cell debris. By using a luciferase kit from Promega, the luciferase activity was detected on a 10-inch luminometer. Each sample was normalized to the protein content. The results were from the average of three independent experiments repeated.

\section{Generation of the in vivo xenograft model}

The Animal experiments were approved by the Ethics Committee of Medical College of Shantou University. To study the impact of SRPK2 on tumorigenesis and growth in vivo, A549 cells were selected (the expression of SRPK2 in A549 cell was the highest) for establishment of a transplant tumor model. Female 6-8 weeks old $\mathrm{BALB} / \mathrm{c}$ nude mice weighing 18-22 g, were purchased from the Guangdong Medical Laboratory Animal Center. The mice were divided into two groups: A549 with shSRPK2 (A549 + sh-SRPK2) and A549 with Scramble (A549 + Scramble), with 6 mice in each group. $3 \times 10^{5}$ cells were subcutaneously injected into the right ventral side. Seven days after the injection, the size of the xenograft tumor was measured by a caliper twice a week, and for the tumor growth curve, the volume was calculated using the formula $\mathrm{V}=\pi / 6 \times$ length $\times$ width $^{2}$. On the $24^{\text {th }}$ day after cell implantation, the experiment was terminated and all mice were euthanized, tumor tissues were extracted, and tumor weight and other parameters were measured. For example, the expression of SRPK2 and Ki-67 in paraffin-embedded tissues were examined. The remaining xenograft tissue was stored at $-80^{\circ} \mathrm{C}$ until use.
Immunofluorescence assays and immunohistochemical staining

Immunohistochemistry assay was used to detect the subcellular localization and protein expression levels of SRPK2 in NSCLC tissue specimens and NSCLC xenograft model. Immediately after surgery, the clinical specimens were fixed in $10 \%$ neutral formalin buffer, embedded in paraffin. The tissues were cut to $5 \mu \mathrm{m}$ sections and then stained by peroxidase (DAB) immunohistochemical or H\&E staining. In simple terms, after proteolytic digestion and peroxidase blockade, tissue slides were incubated with anti-SRPK2 primary antibody overnight at a $1: 150$ dilution at $4^{\circ} \mathrm{C}$. After washing the tissue sections, the protein staining was observed by peroxidaseconjugated secondary antibody and staining original substrate.

SKMES1 and CALU3 (the expression of SRPK2 in these two cells is the same), NCHI1573 and A549 cells (the expression of SRPK2 in both cells is relatively high) were plated into a 24 -well plate containing coverslips. Cells were transfected with overexpressed SRPK2 plasmid and SRPK2
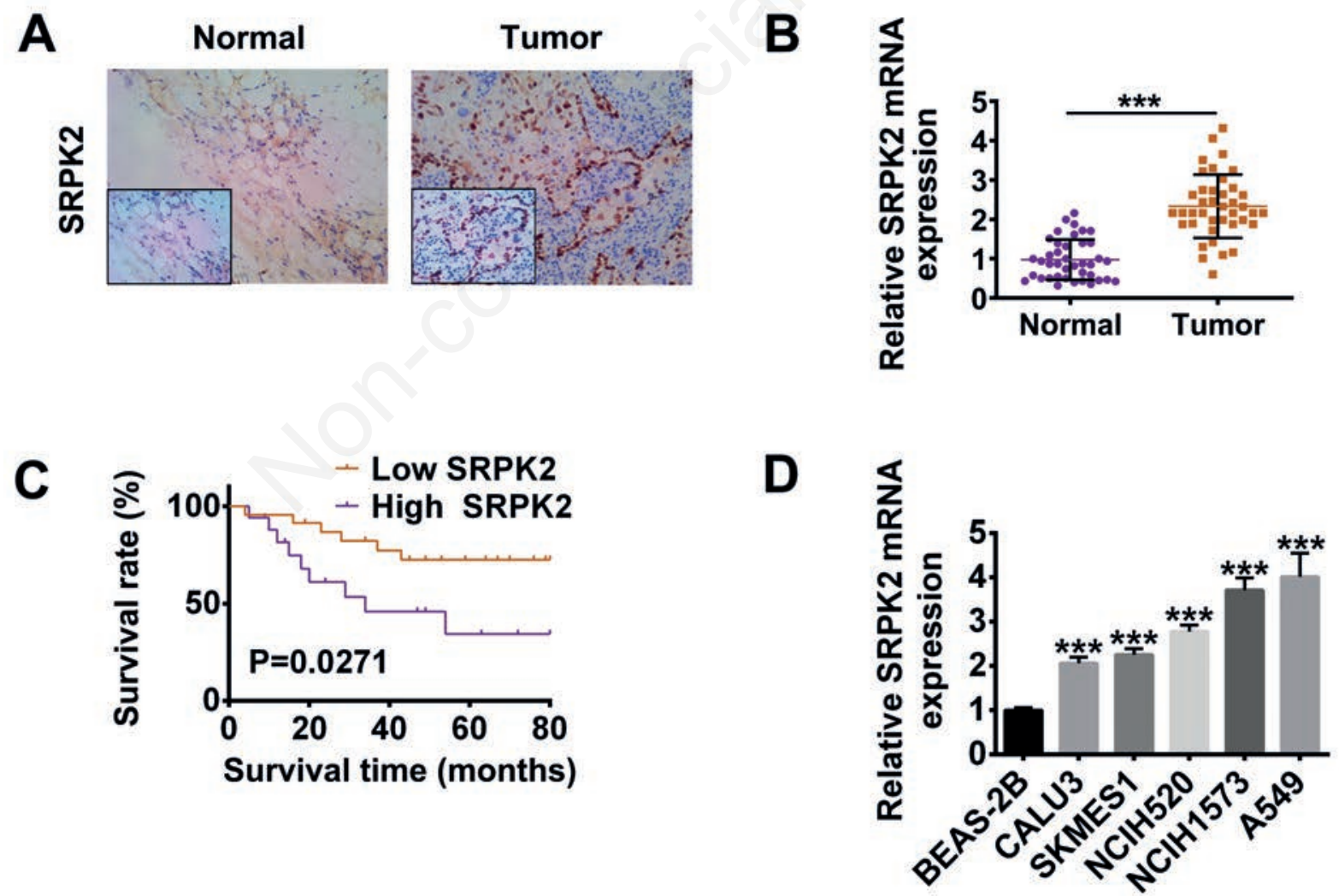

Figure 1. SRPK2 are overexpressed in NSCLC tissues and cell lines and its association with overall survival of NSCLC patients. A) SRPK2 expression assessed using immunostaining in NSCLC tissues; magnification $\times 40$; insert, magnification $\times 200$. B) $q R T-P C R$ analysis of the SRPK2 expression in NSCLC tissues. C) Kaplan-Meier survival graphs for overall survival (OS) of patients with NSCLC $(P=0.0271)$. D) SRPK2 expression was analyzed using $q R T-P C R$ in five NSCLC cell lines. The data were presented as mean \pm SD. ${ }^{*} \mathbf{P}<\mathbf{0 . 0 5},{ }^{* *} \mathbf{P}<\mathbf{0 . 0 1}$ compared with Normal group. 
shRNA and cells treated with DMSO and RPMI medium were used as controls. Then, cells were first fixed with $4 \%$ paraformaldehyde/Hepes for $20 \mathrm{~min}$ and washed with PBS, the cells were permeabilized for 20 min in $1 \%$ Triton X-100/PBS, washed with PBS and blocked with 3\% BSA / PBS-T (PBS containing $0.05 \%$ Tween-20) for 30 min. Cells were incubated with primary antibodies against SRPK2 overnight at a 1:1000 dilution at $4^{\circ} \mathrm{C}$. After washing, the cells were incubated with anti-mouse-IgGAlexa fluorescein 488 (Invitrogen) secondary antibody for $2 \mathrm{~h}$. The nuclear was counterstained with 4',6-diamidino-2-phenylindole (DAPI, Invitrogen).
Image acquisition was performed using an inverted fluorescence microscope (EVOS FL, Thermo Fisher Scientific, Waltham, MA, USA).

\section{Statistical analysis}

All data in the experiment are expressed as the mean standard error of the three experimental means. Student's $t$-test was used to analyze unpaired data. Multiple sets of data were compared using one-way analysis of variance (GraphPad ver. 7.0, GraphPad Software, CA, USA). Survival rates during the 120-day observation period were assessed using the Kaplan-Meier test. The difference was statistically significant at $\mathrm{P}<0.05$.

\section{Results}

SRPK2 is overexpressed in NSCLC tissues and cell lines and is associated with overall survival of NSCLC patients

Firstly, immunohistochemistry was used to analyze the expression of SRPK2 in a series of 60 NSCLC tissues and adjacent normal lung tissues. Compared with the corresponding adjacent tissues, the expression level of SRPK2 in NSCLC tissues was significantly increased (Figure 1A). The SRPK2 expression levels in NSCLC cell lines and tissues were detected by qRT-
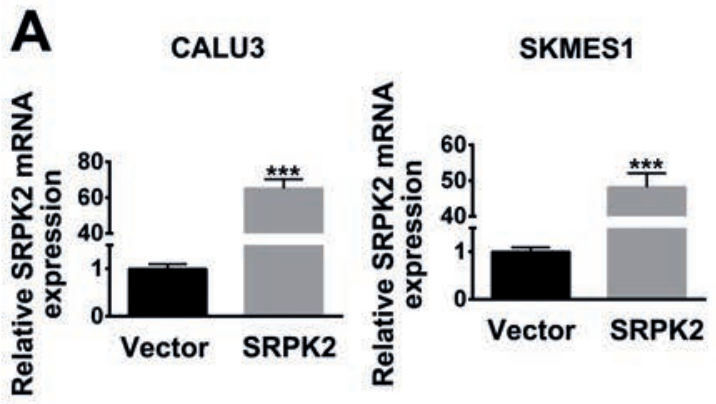

B

Vector SRPK2
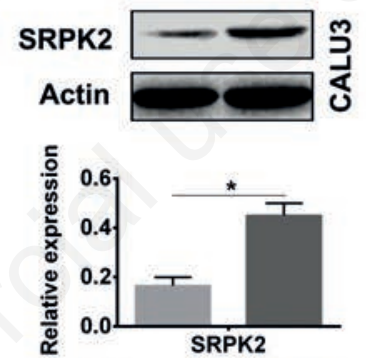

D
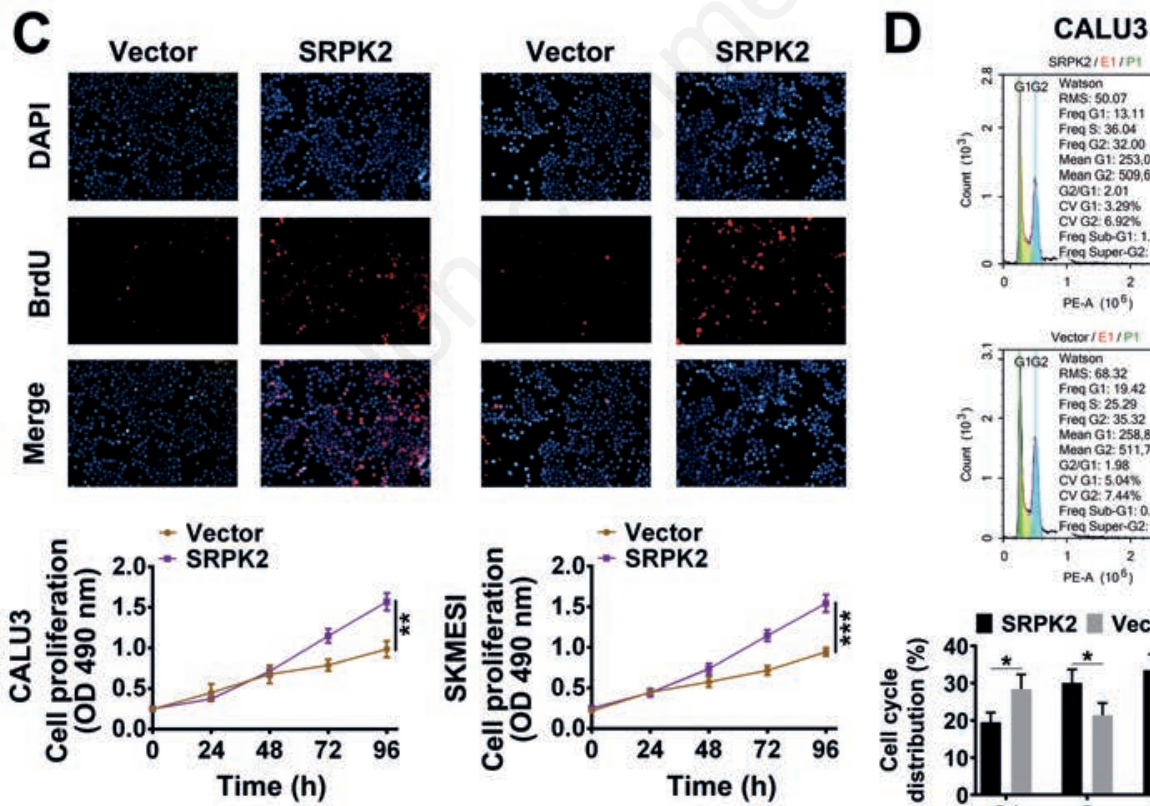
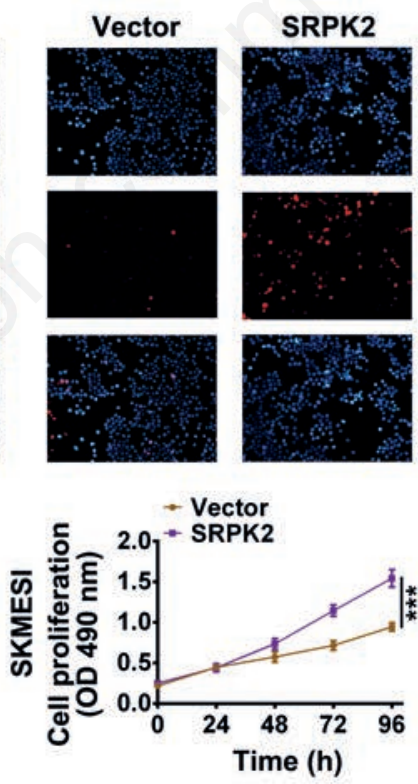
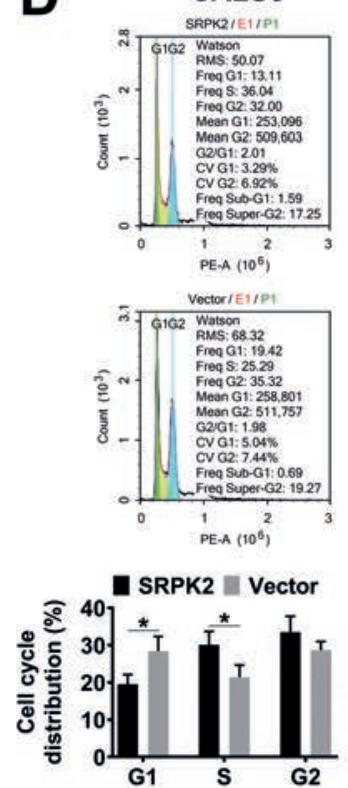

Vector SRPK2
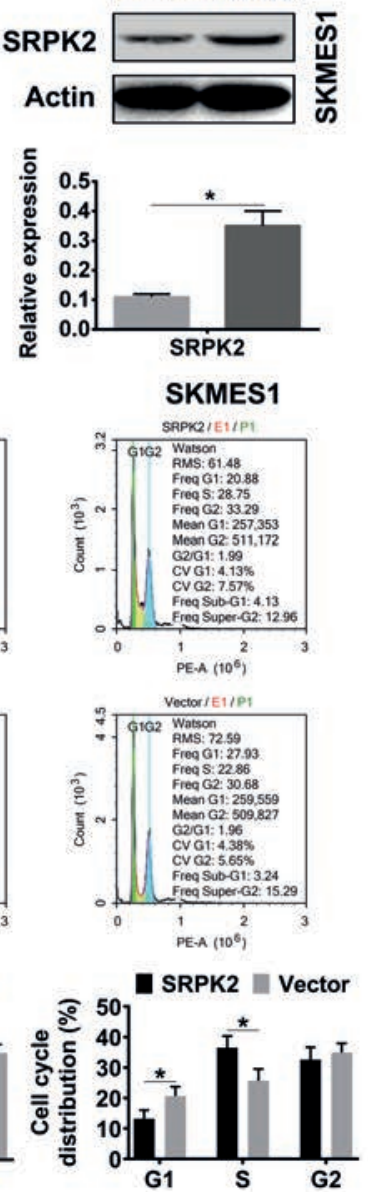

Figure 2. Overexpression of SRPK2 promotes NSCLC cell proliferation and cell cycle progression. Over-expression of SRPK2 and empty vector (Vector) were transfected in CALU3 and SKMES1 cells. A) qRT-PCR analysis of the SRPK2 mRNAs expression level. (B) Western blot analysis of the SRPK2 proteins expression level; actin was used as a loading control, a densitometric analysis of the specific signals was performed using ImageJ software $\left({ }^{*} \mathrm{P}<0.05\right)$. C) The effect of SRPK2 on cell proliferation in vitro using BrdU, SRPK2 overexpression were significantly increased when compared with the Vector at BrdU staining cells and different points in time $($ all $P<0.05)$. D) FACS detection in vitro showed a significant decrease of $G_{1}$ cells in the over-expression of SRPK2 groups, correspondingly increased the $S$ phase cells. 
PCR. The results showed that SRPK2 expression was significantly higher in NSCLC tissues than in normal tissue (Figure 1B). Next, we assessed the expression of SRPK2 in five lung cancer cell lines (A549, CALU3, SKMES1, NCIH520 and NCIH1573), and the expression of SRPK2 in NSCLC cell lines was significantly increased compared with human lung epithelial cells BEAS-2B. (Figure 1D). Analysis of SRPK2 was performed by Kaplan-Meier method and comparison of log-rank test to determine the clinical significance of SRPK2 in NSCLC. SRPK2 mRNA expression predicted a poor prognosis for overall survival in patients with NSCLC, and patients with high expression of SRPK2 had a much shorter median overall survival time than those with low expression. In patients with NSCLC, survival curves of high and low expression of SRPK2 had remarkable differences in overall survival ( $\mathrm{P}=0.0271)$ (Figure 1C).

\section{Overexpression of SRPK2 promotes} NSCLC cell proliferation and cell cycle progression

We transfected CALU3 and SKMES1 cells with the overexpression SRPK2 plasmid and the empty vector (Vector) for the corresponding negative control. The expression of SRPK2 was detected by qRT-PCR and Western blot. In the CALU3 and SKMES1 cell lines transfected with overexpression plasmid, the SRPK2 mRNA and protein levels were significantly increased in the comparison with the Vector group, and the difference was statistically significant, indicating that the transfection efficiency was successfully established (Figure 2 A-B). To explore whether SRPK2 was involved in cell proliferation, we performed BrdU incorporation assay. After $48 \mathrm{~h}$ of transfection, overexpression SRPK2 cells transfected with CALU3 and SKMES1 were notably increased compared with the Vector group, indicating that SRPK2 overexpression promoted cell proliferation (Figure 2C). In addition, flow cytometry results showed that overexpression of SRPK2 promoted cell cycle progression by decreasing the proportion of G1 phase and increasing the number of $\mathrm{S}$ phase cells in SKMES1 and CALU3 cell lines compared to the Vector group. These results indicate that SRPK2 promotes cell transition from $\mathrm{G}_{1}$ to $\mathrm{S}$ phase and promotes cell cycle progression (Figure g 2D).
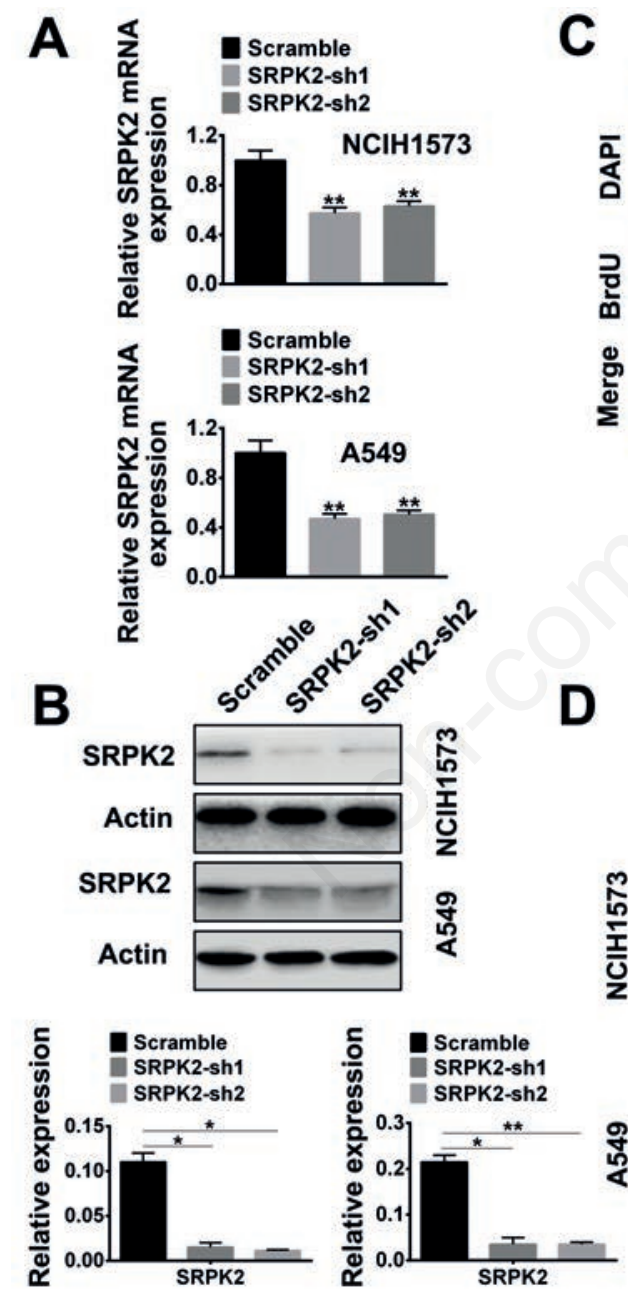
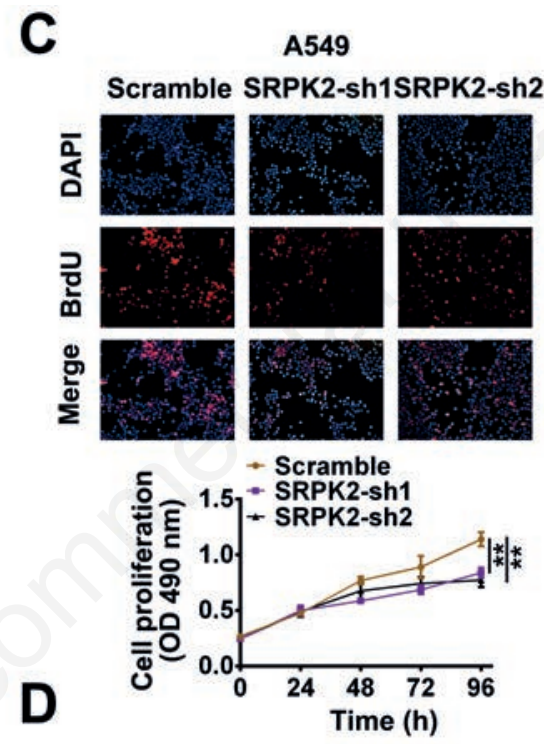

NCIH1573

Scramble SRPK2-sh1 SRPK2-sh2
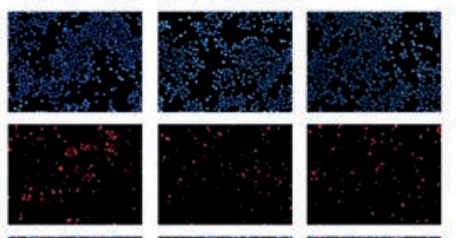

a
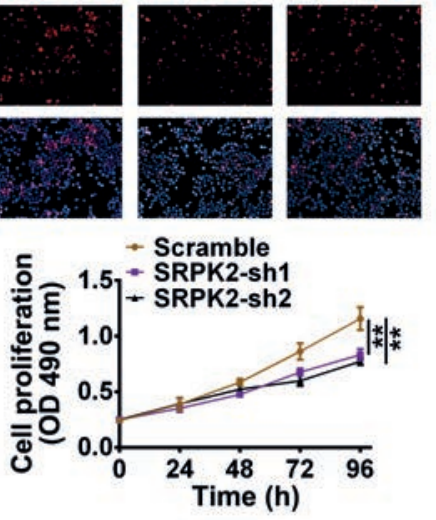
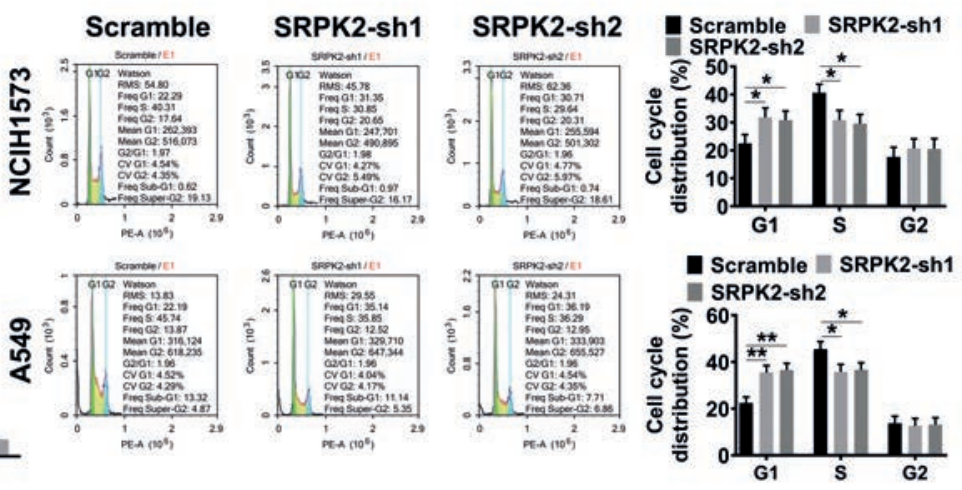

Figure 3. Knockdown of SRPK2 inhibits NSCLC cell proliferation and cell cycle arrest. SRPK2-sh1 and SRPK2-sh2 and their mismatch siRNA (Scramble) were constructed to transfect in the NCIH1573 and A549 cells. A) qRT-PCR analysis of the SRPK2 mRNAs expression level. B) Western blot analysis of the SRPK2 proteins expression level. Actin was used as a loading control, a densitometric analysis of the specific signals was performed using ImageJ software $(* \mathrm{P}<0.05)$. C) Effect of SRPK2 on cell proliferation in vitro using BrdU; the proportion of BrdU-stained cells decreased and cell proliferation decreased in in the SRPK2 silenced groups (all P<0.05). D) FACS detection in NCIH1573 and A549 cells, sh-SRPK2 downregulation dramatically increased in $G_{1}$ cells and the proportion of $S$ phase decreased. 


\section{Knockdown of SRPK2 inhibits NSCLC cell proliferation and cell cycle arrest}

Two shRNAs (SRPK2-sh1 and SRPK2sh2) with SRPK2 targeted inhibition and their mismatched siRNA (Scramble) were transfected into NCIH1573 and A549 cells. Afterward, the inhibitory effects were detected d by qRT-PCR and Western blot, and the results showed that the expression of SRPK2 mRNA in sh-SRPK2 A549 and NCIH1573 cells was significantly decreased (Figure 3A). Correspondingly, the protein profiles of SRPK2 in sh-SRPK2 A549 and NCIH1573 cells were also significantly decreased (Figure 3B). BrdU assay displayed that the cell proliferation ability was decreased and the proportion of BrdU stained cells was decreased (Figure 3B, top). In addition, within $24 \mathrm{~h}$ after the initial sowing period, the proliferation rates of the two SRPK2-silencing A549 and NCIH1573 cells were substantially the same, however from the $48^{\text {th }}$ hour to the end, their proliferation rate was remarkably inhibited (Figure 3B, bottom). By analyzing cell cycle distribution by flow cytometry (Figure 3D), we found that down-regulation of sh-SRPK2 dramatically increased the number of A549 and NCIH1573 cells in $\mathrm{G}_{1}$ phase, and the proportion of $\mathrm{S}$ phase cells was decreased, indicating that down-regulation of SRPK2 leads to cell cycle arrest.

\section{SRPK2 promotes $E 2 F 1$ transcrip- tion of downstream cell cycle-related genes}

Gain and loss of function assays were conducted by transfection of overexpression of SRPK2 plasmid and Vector into CALU3 and SKMES1 cells, and SRPK2sh1, SRPK2-sh2 and Scramble into the NCIH1573 and A549 cells respectively. The expression of cyclinE1, p45 ${ }^{\text {skp2 }}$ and E2F1 was examined by qRT-PCR and Western blot. Our results revealed that the mRNA and protein levels of CyclinE1 and p45 skp2 expression were remarkably increased, and the change of E2F1 was not obvious compared with Vector group in the CALU3 and SKMES1 cell lines. However, compared with the Scramble, the expression of CyclinE and $\mathrm{p} 45^{\text {skp2 }}$ was decreased after knockdown of SRPK2, and the change of E2F1 was not significant in the NCIH1573 and A549 cells (Figure 4 A,B).

Overexpression of E2F1 was performed by transfection in HEK-293T cells. E2F1 expression was confirmed by qRTPCR and Western blot. Our results revealed that the mRNA of E2F1 expression significantly increased (Figure 4C) and the protein level of E2F1 expression also expressively increased (Figure 4D).

Luciferase assay was carried out by transiently transfecting a dual luciferase reporter vector under the control of the Cyclin E (CyclinE-luc) or p45 ${ }^{\text {SKP2 }}$ (Skp2luc) promoter 1 in HEK-293T cells. The luciferase activity was measured $48 \mathrm{~h}$ posttransfection. The result showed that luciferase activity of Cyclin E1 and p45 $5^{\text {skp2 }}$ was increased after overexpression of SRPK2, while the luciferase activity of CyclinE1 and $\mathrm{p} 45^{\text {skp2 }}$ was decreased after knockdown of SRPK2 (Figure 4E).

\section{SC35 promotes transcriptional regu- lation of downstream cell cycle- related genes by $E 2 F 1$}

Overexpression of SC35 and pcDNA3.1 were transfected into CALU3 and SKMES1 cells, and siRNA, siSC35 and Scramble were transfected into the NCIH1573 and A549 cells. SC35 expression was confirmed by qRT-PCR and Western blot. Our results showed that the mRNA and protein level of SC35 expression was remarkably increased in the CALU3 and SKMES1 cell lines. However, comparing with the Scramble, the expression of SC35 was decreased after knockdown in the NCIH1573 and A549 cells (Figure 5 A,B). Cyclin E1, p45 ${ }^{\text {skp2 }}$ and E2F1 expression was confirmed by qRT-PCR and Western blot. Our results proved that in the CALU3 and SKMES1 cell lines, the mRNA and protein levels of Cyclin E1 and p45 skp2 were significantly increased in SC35 overexpression group compared with the pcDNA3.1 group, while the expression of E2F1 was not significantly changed indicating that SC35 can regulate the expression of Cyclin E1 and p45 skp2 without affecting the level of E2F1. However, comparing with the Scramble, the expression of Cyclin E and $\mathrm{p} 45^{\mathrm{skp} 2}$ was decreased after SC35 knockdown, and the change of E2F1 was not significant in the NCIH1573 and A549 cells (Figure $5 \mathrm{C}, \mathrm{D}$ ). These results suggest that $\mathrm{SC} 35$ can regulate Cyclin E and p45 skp2 expression without affecting E2F1 levels.

HEK-293T cells were co-transfected with overexpression of E2F1plasmid and overexpressed or knockdown SC35 plasmids. Transiently transfected luciferase in HEK-293T cells expressed a dual luciferase reporter vector under the control of the Cyclin E (Cyclin E-luc) or p45 ${ }^{\mathrm{SKP} 2}$ (Skp2luc) promoter. The luciferase activity was measured $48 \mathrm{~h}$ post-transfection. The result showed increased Cyclin E1 and p45 skp2 luciferase activity after overexpression of SC35, and decreased Cyclin E1 and p45 $5^{\text {skp2 }}$ luciferase activity after knockdown of SC35 (Figure 5E).
SRPK2 mediates E2F1 expression by phosphorylating SC35 and promotes transcriptional regulation of downstream cell cycle-related genes

Overexpression of SRPK2 plasmid and Vector, SRPK2-shRNA and Scramble were transfected into CALU3, SKMES1 cells, NCIH1573 and A549 cells. SC35 and phosphorylated-SC35 (p-SC35) expression was confirmed by Western-blot. Our results proved that the protein level of p-SC35 expression was remarkably increased in the CALU3 and SKMES1 cell lines. However, the expression of p-SC35 was decreased after knockdown in the NCIH1573 and A549 cells, while the change of SC35 expression was not significantly in the four NSCLC cell lines (Figure 6A). SRPK2 phosphorylated SC35, whereas mutant SRPK2 ${ }^{\text {T492A }}$ had a weak ability to phosphorylate $\mathrm{SC} 35$.

Overexpression of SRPK2 $2^{\mathrm{T} 492 \mathrm{~A}}$ and pcDNA3.1 were transfected in CALU3 and SKMES1 cells. SRPK2 ${ }^{\mathrm{T} 492 \mathrm{~A}}$ expression was confirmed by qRT-PCR and Western blot. The results showed that the mRNA and protein level of SC35 expression was remarkably increased in the CALU3 and SKMES1 cell lines (Figure 6 B,C).

Overexpression of SRPK2 and overexpression of SRPK2 $2^{\mathrm{T} 492 \mathrm{~A}}$ was co-transfected with overexpressing SC35 plasmid in CALU3, and SKMES1 cells, respectively. $\mathrm{SC} 35$ and p-SC35 expression was confirmed by Western-blot. Our results showed that the protein level of $\mathrm{p}$-SC35 expression was significantly increased in the CALU3 and SKMES1 cell lines (Figure 6D). Overexpression of SRPK2 increased SC35 phosphorylation, while SC35 phosphorylation was further enhanced after overexpression of SC35. However, SRPK2 ${ }^{\text {T492A }}$ did not increase SC35 phosphorylation. CyclinE1, p45 skp2 and E2F1 expression was confirmed by qRT-PCR and Western blot. The results presented that overexpression of SRPK2 up-regulated the expression of Cyclin E and $\mathrm{p} 45^{\text {skp2 }}$, while overexpression of SC35, further increased the expression of Cyclin E and p45 skp2. While, SRPK2 ${ }^{\mathrm{T} 492 \mathrm{~A}}$ could not increase the expression of Cyclin E and $\mathrm{p} 45^{\mathrm{skp} 2}$ (Figure $6 \mathrm{E}, \mathrm{F}$ ).

\section{Knockdown of SRPK2 inhibits tumor growth in vivo}

To reveal whether SRPK2 plays a role in the growth of NSCLC in vivo, we used A549 cells (A549 + sh1-SRPK2) to establish a xenograft mouse model to examine the effect of SRPK2 on down-regulating tumorigenicity in vivo. The tumor growth curve showed that tumor formation was significantly slower in the SRPK2 knock- 
A
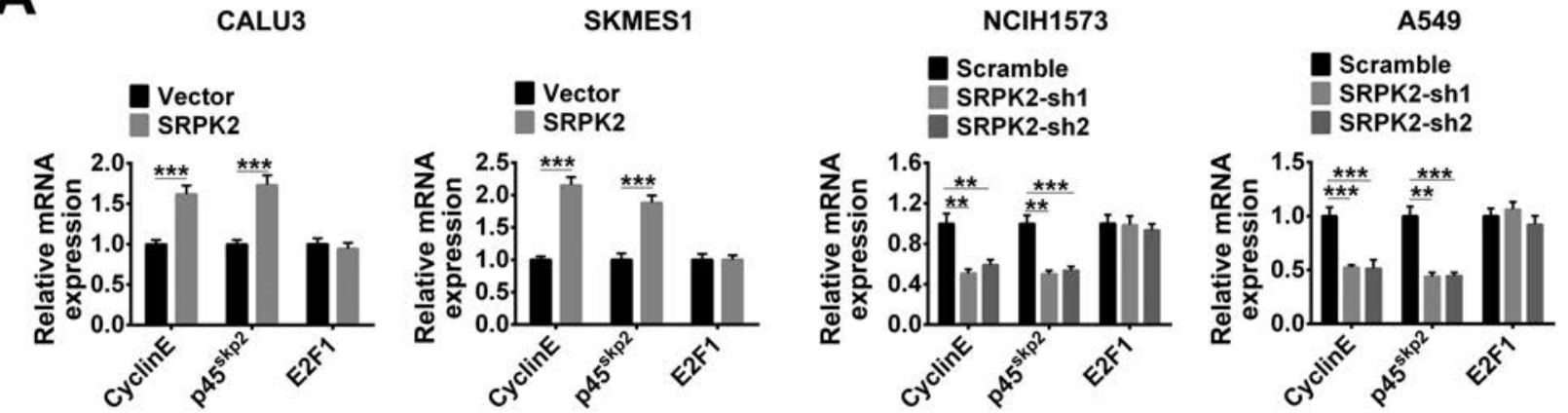

B
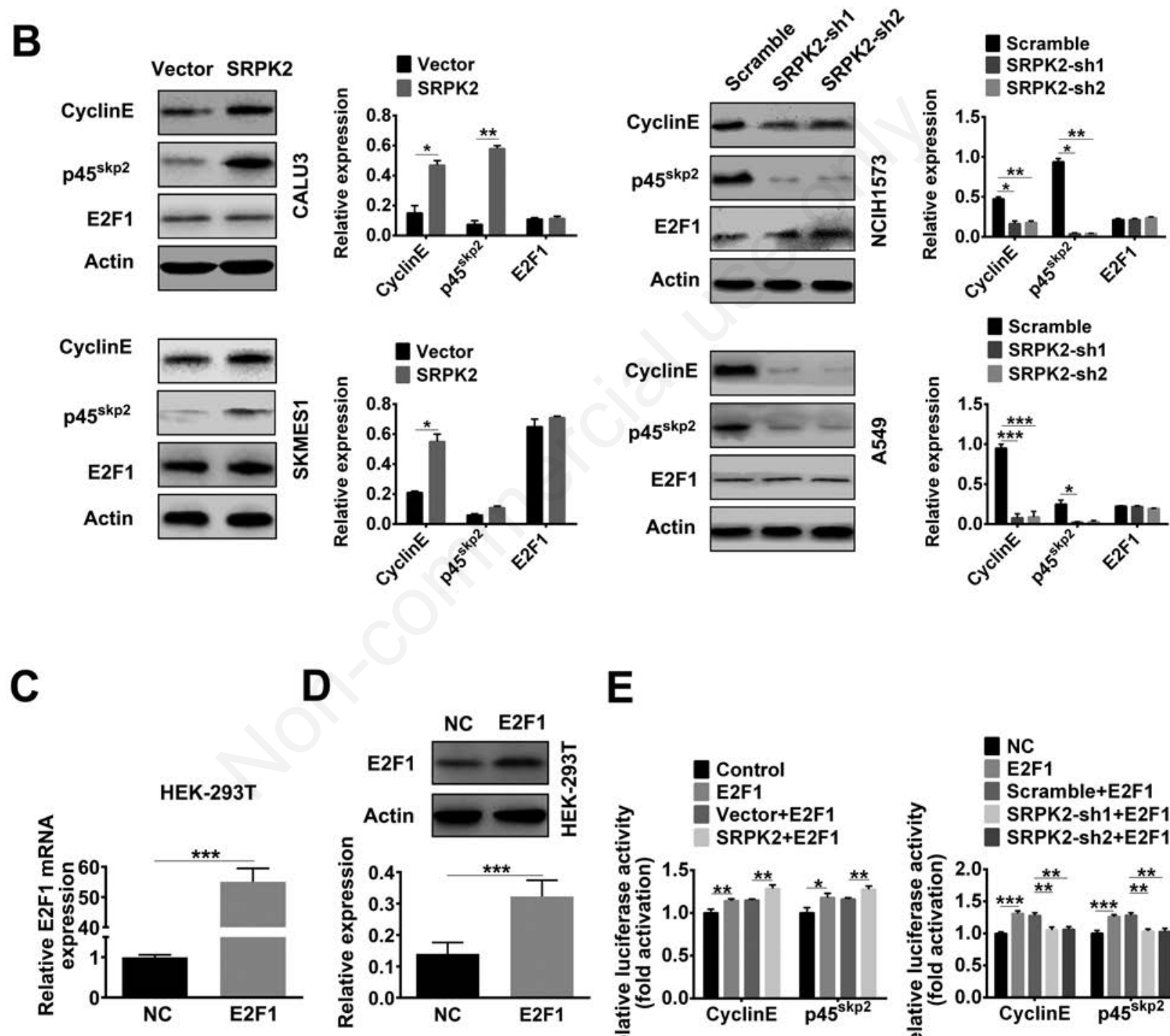

E
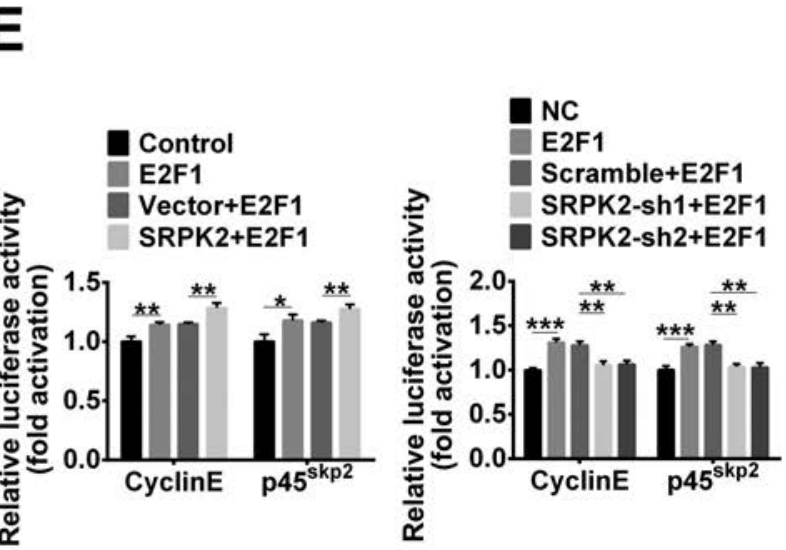

Figure 4. SRPK2 promotes E2F1 transcription of downstream cell cycle-related genes. Overexpression of SRPK2 and Vector were transfected in CALU3 and SKMES1 cells, SRPK2-sh1, SRPK2-sh2 and Scramble were transfected in the NCIH1573 and A549 cells. A) qRTPCR analysis of the Cyclin E1, p45 skp2 and E2F1 mRNAs expression level. B) Western blot analysis of the Cyclin E1, p45 ${ }^{\text {skp2 }}$ and E2F1 proteins expression level. Actin was used as a loading control, a densitometric analysis of the specific signals was performed using ImageJ software $\left.\left({ }^{*} \mathrm{P}<0.05,{ }^{* *} \mathrm{P}<0.01\right) . \mathrm{C}\right) \mathrm{qRT}-\mathrm{PCR}$ analysis of the E2F1 mRNAs expression level in overexpression of E2F1. D) Western blot analysis of the E2F1 proteins expression level in overexpression of E2F1. E) Dual luciferase reporter assays the luciferase activity of Cyclin E1 and p45 $5^{\text {sk2 }}$ when overexpression of E2F1 treated HEK-293T cells and then overexpression or knockdown of SRPK2. 
out group (Figure 7 A-C). We also found a significantly reduction in $\mathrm{Ki}-67$ and SRPK2-positive nuclear expression cells in sh-SRPK2 group compared with Scramble (Figure 7D).

\section{Discussion}

Lung cancer is one of the most common cancers and the leading cause of cancerrelated death. ${ }^{24}$ NSCLC accounts for $75 \%$ of lung cancer, and due to its complicated pathological mechanism, the current knowledge is still limited. ${ }^{25}$ The high mortality rate of NSCLC with a rapid growth trend is a challenge and has gradually become the focus of public opinion and a major burden on human health. ${ }^{26}$ It is reported for the first
A
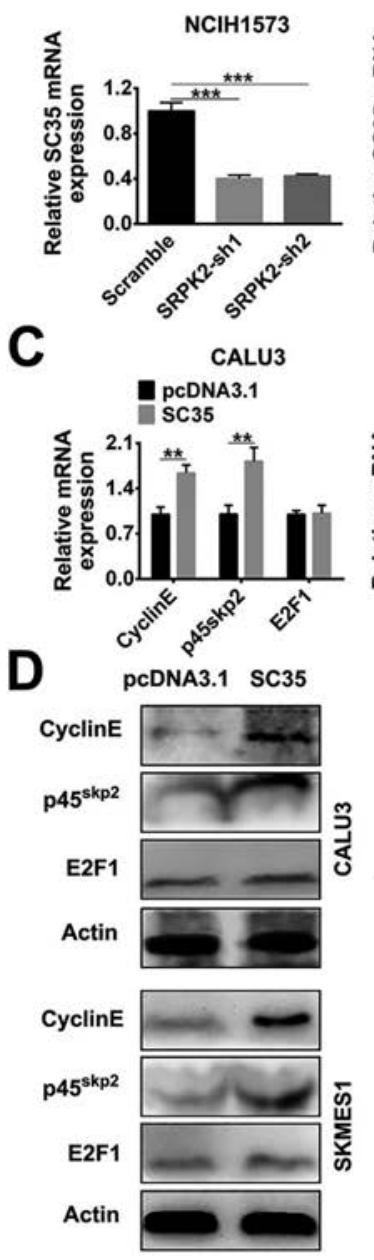

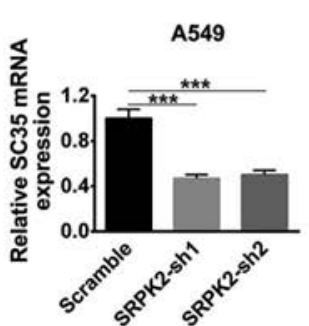

SKMES1
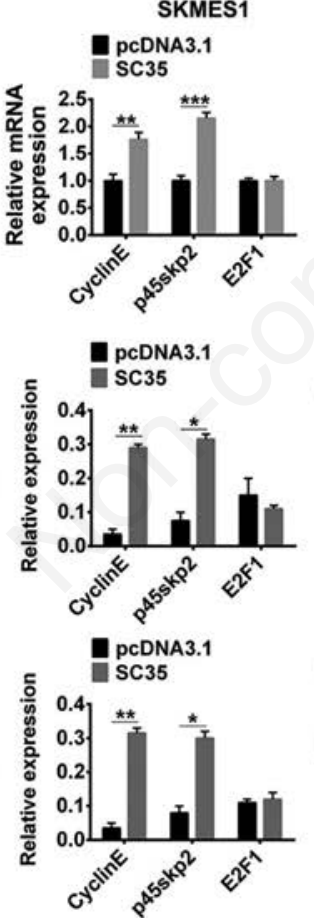

B
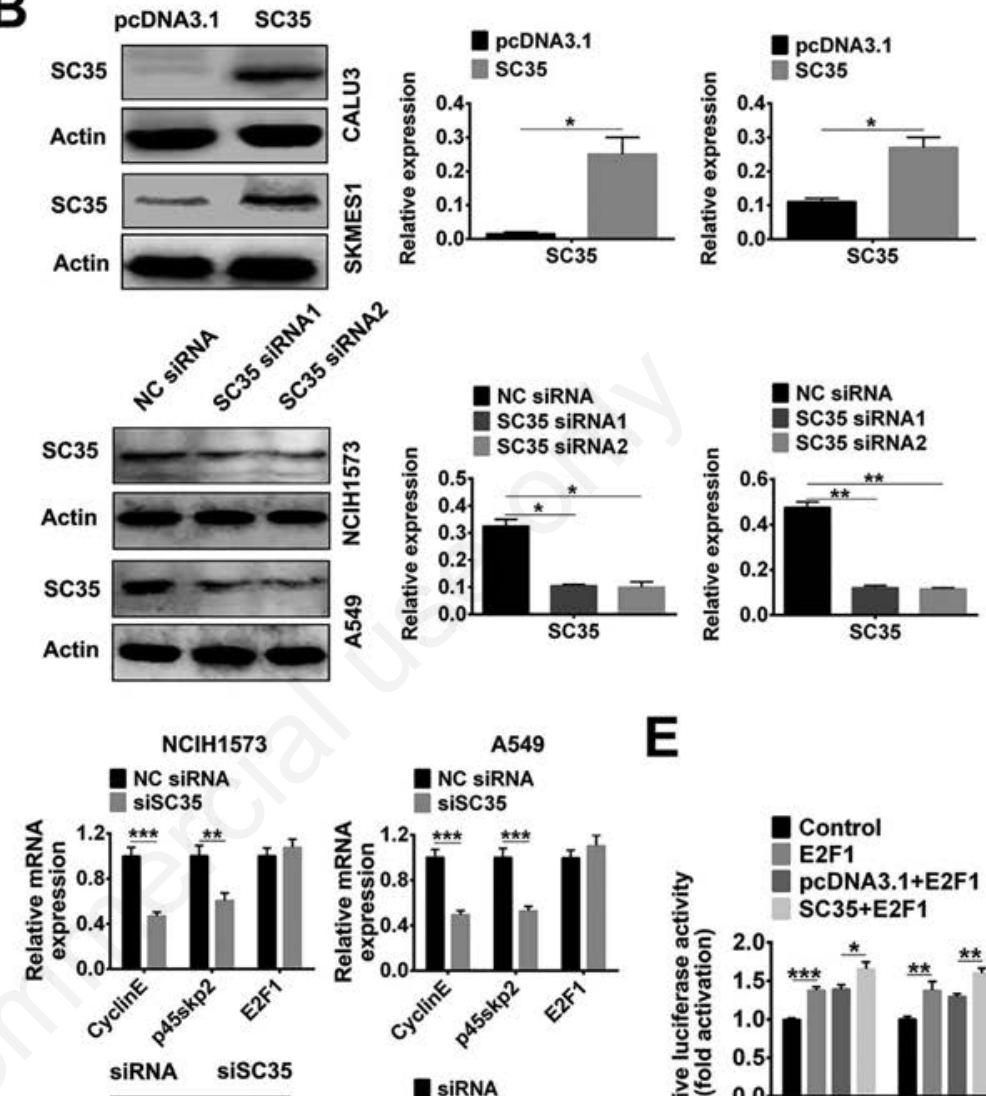

$\mathbf{E}$
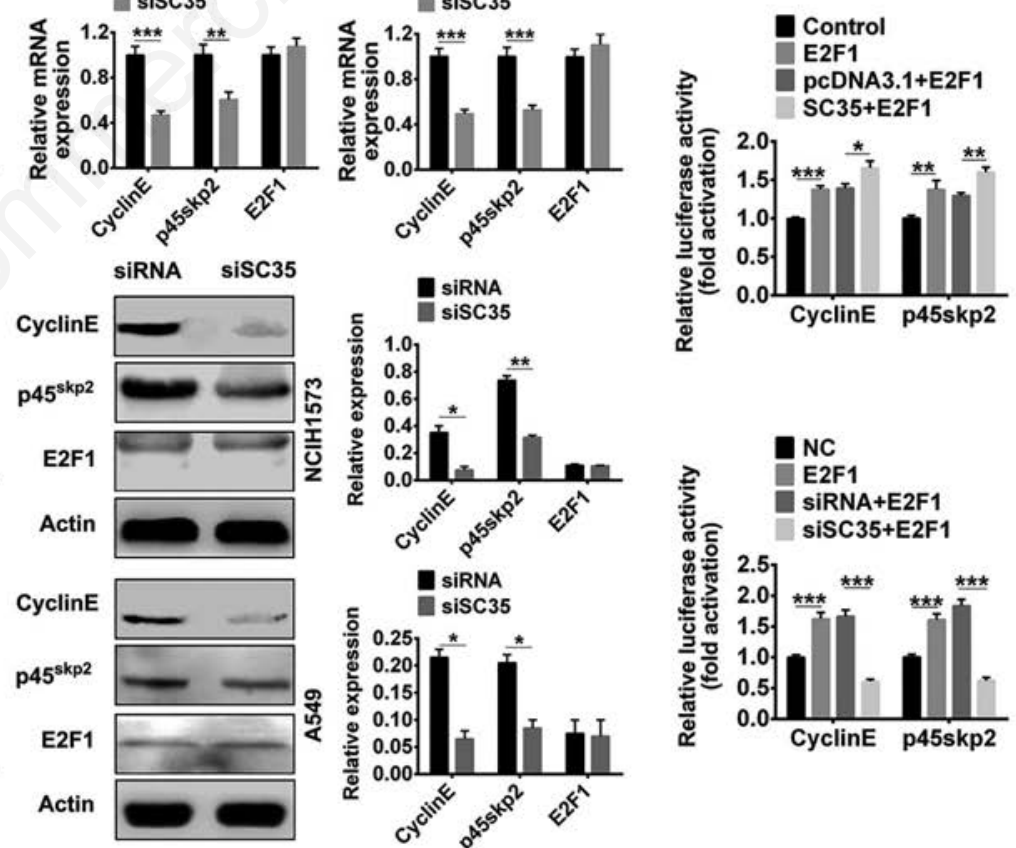

Figure 5. SC35 promotes transcriptional regulation of downstream cell cycle-related genes by E2F1. Overexpression of SC35 and pcDNA3.1 were transfected in CALU3 and SKMES1 cells, siRNA, siSC35 and Scramble were transfected in the NCIH1573 and A549 cells. A) qRT-PCR analysis of the SC35 mRNAs expression level in four NSCLC cell lines. B) Western blot analysis of the SC35 proteins expression level in four NSCLC cell lines; actin was used as a loading control, a densitometric analysis of the specific signals was performed using ImageJ software $\left.\left({ }^{*} \mathrm{P}<0.05,{ }^{* *} \mathrm{P}<0.01\right) . \mathrm{C}\right)$ qRT-PCR analysis of the Cyclin E1, $\mathrm{p} 45^{\text {skp2 }}$ and E2F1 mRNAs expression level in four NSCLC cell lines. D) Western blot analysis of the Cyclin E1, p45 $5^{\text {skp2 }}$ and E2F1 proteins expression level in four NSCLC cell lines; actin was used as a loading control, a densitometric analysis of the specific signals was performed using ImageJ software $\left({ }^{*} \mathrm{P}<0.05\right.$, $\left.{ }^{* *} \mathrm{P}<0.01\right)$. E) Dual luciferase reporter assay; the luciferase activity of Cyclin E1 and $\mathrm{p} 45^{\text {skp } 2}$ when overexpression of E2F1 treated HEK293 T cells and then overexpression or knockdown of SC35. 
time that the expression of SR phosphorylation kinases SRPK1 and SRPK2 were upregulated in NSCLC. ${ }^{16}$ In our study, it was found that the expression of SRPK2 in NSCLC tissues was significantly higher than that of corresponding non-tumor tissues. The survival rate of patients with high SRPK2 expression was significantly lower than that of patients with low expression of SRPK2. However, the role of SRPK2 in the progression of NSCLC remains unclear.

SRPK is a family of cell cycle regulatory kinases, and it phosphorylates nuclear speckles containing a protein-containing serine/arginine (SR) domain that mediates pre-mRNA splicing. ${ }^{9,27}$ The pre-mRNA splicing independent function of SRPK2 plays a pivotal role in cell cycle progression and apoptosis. ${ }^{28}$ SRPK2 silenced cells accumulate in the $G_{1}$ phase. However, overexpression of SRPK2 results a large increase in the $\mathrm{G}_{2}-\mathrm{M}$ transition. ${ }^{12,13}$ SRPK2 controls the expression of cell cycle regulators including cyclin B1, cyclin D1 and CDK4 by phosphorylation of SC35. ${ }^{12} \mathrm{SC} 35$ is classified as a SR family protein and is the most key category of splicing regulatory proteins. ${ }^{29}$ In the process of controlling apopto- sis, the first functional link between the transcription factor E2F1 and a component of the splicing machinery SC35 was found..$^{30}$ During the development of the cell cycle, the content of SRSF2 protein and mRNA is the same as that of E2F1, which rises sharply from $G_{1}$ to $S$, and continues to rise as the cells enter $S$ phase. ${ }^{22}$ In our study, we constructed the CALU3 and SKMES1 cell lines overexpressing of SRPK2 and SRPK2 silenced NCIH1573 and A549 cell lines by transfection of SRPK2-shRNA. In vitro BrdU assay results demonstrated that SRPK2 affects the proliferation of NSCLC
A
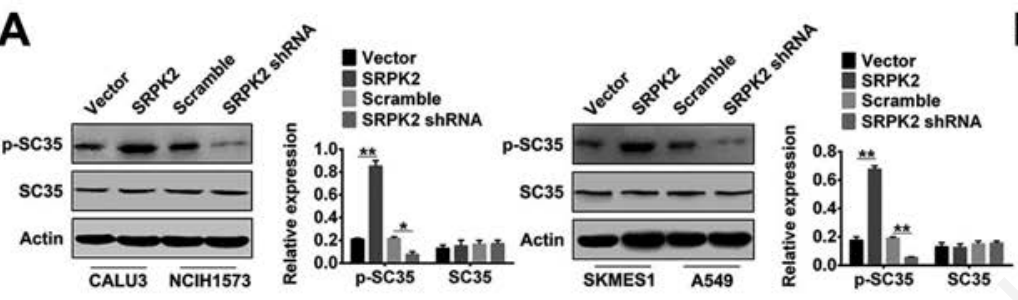

B
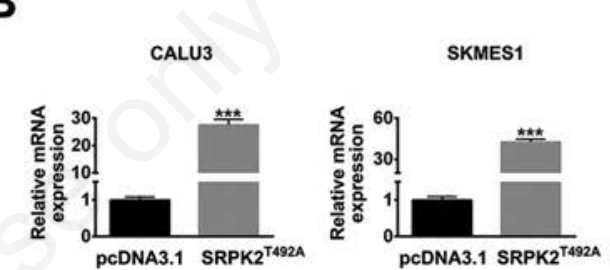

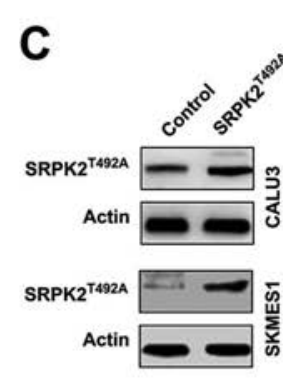

E

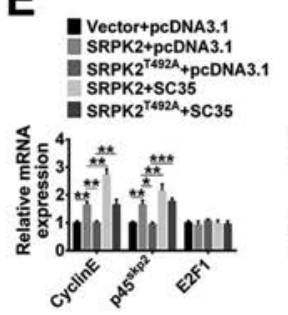

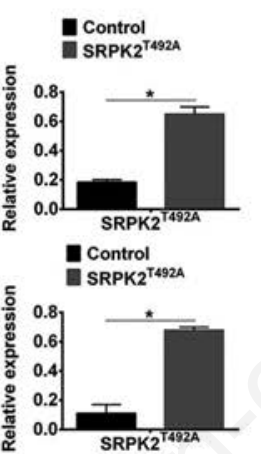

D
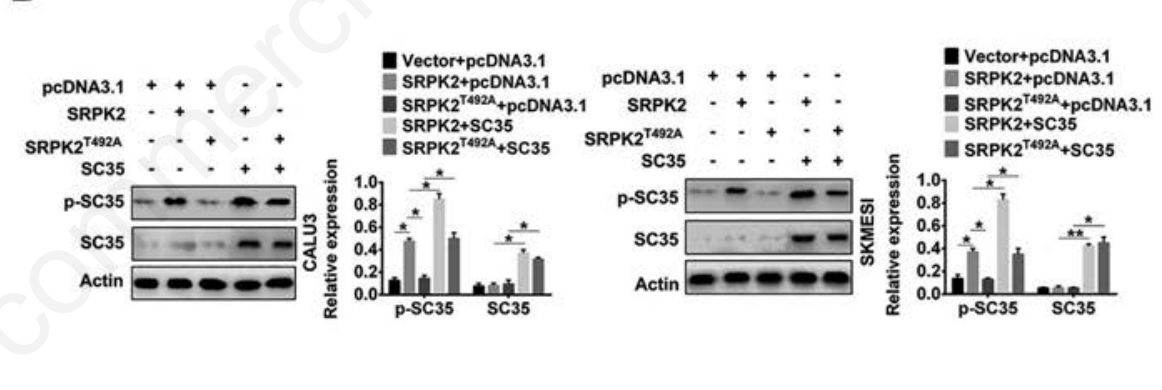

F

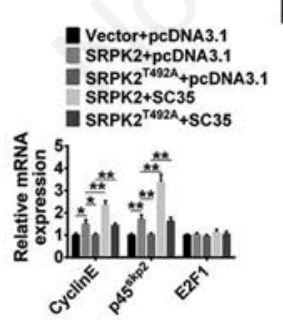

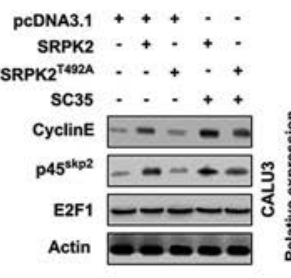

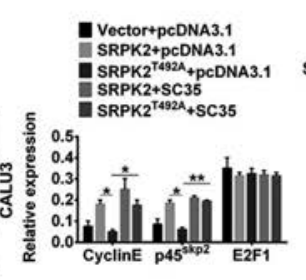

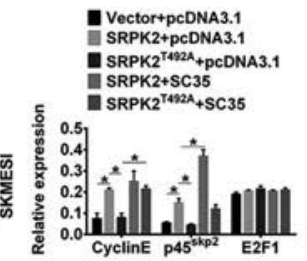

Figure 6. SRPK2 mediates E2F1 expression by phosphorylating SC35 and promotes transcriptional regulation of downstream cell cyclerelated genes. Overexpression of SRPK2 and Vector, SRPK2-shRNA and Scramble were transfected in CALU3, SKMES1 cells, NCIH1573 and A549 cells. A) Western blot analysis of the SC35 and p-SC35 proteins expression level in four NSCLC cell lines; actin was used as a loading control, a densitometric analysis of the specific signals was performed using Image $\mathrm{J}$ software $\left({ }^{*} \mathrm{P}<0.05,{ }^{* *} \mathrm{P}<0.01\right)$. B) qRT-PCR analysis of the SRPK2 ${ }^{\mathrm{T} 492 \mathrm{~A}}$ mRNAs expression level in CALU3 and SKMES1 cell lines. C) Western blot analysis of the SRPK2 ${ }^{\text {T492A }}$ proteins expression level in CALU3 and SKMES1 cell lines. Actin was used as a loading control, a densitometric analysis of the specific signals was performed using ImageJ software $\left({ }^{*} \mathrm{P}<0.05,{ }^{* *} \mathrm{P}<0.01\right)$. D) Western blot analysis of the SC35 and $\mathrm{p}-\mathrm{SC} 35$ proteins expression level in CALU3 and SKMES1 cell lines when over-expression of SRPK2 and over-expression of SRPK2 ${ }^{\mathrm{T} 492 \mathrm{~A}}$ co-transfection of CALU3, and SKMES1 cells with overexpressing SC35 plasmid. E) qRT-PCR analysis of the Cyclin E1, p45 ${ }^{\text {skp2 }}$ and E2F1 mRNAs expression level in in CALU3 and SKMES1 cell lines when over-expression of SRPK2 and over-expression of SRPK2 ${ }^{\mathrm{T} 492 \mathrm{~A}}$ cotransfection of CALU3, and SKMES1 cells with overexpressing SC35 plasmid. F) Western blot analysis of the Cyclin E1, p45sk2 and E2F1 protein expression level in CALU3 and SKMES1 cell lines when over-expression of SRPK2 and over-expression of SRPK2 ${ }^{\mathrm{T} 492 \mathrm{~A}}$ co- $^{-}$ transfection of CALU3, and SKMES1 cells with overexpressing SC35 plasmid. 
cells. Overexpression of SRPK2 promotes NSCLC cell proliferation. On the other side, flow cytometry assay showed that SRPK2 also affected the progression of NSCLC cell cycle. The results showed that knockdown of SRPK2 inhibited NSCLC cell proliferation and cell cycle arrest, while
SRPK2 overexpression promotes the proliferation and cell cycle progression of NSCLC cells by affecting the regulation of downstream cell cycle-related gene E2F1 transcription. Our study also showed that SRPK2 regulates transcription of downstream cell cycle-related genes by E2F1 by phosphorylation of SC35. SC35 regulates Cyclin $\mathrm{E}$ and $\mathrm{p} 45^{\mathrm{skp} 2}$ expression, which in turn affects cell cycle progression. In an in vitro experiment of xenograft mouse model, after knocking down SRPK2, the volume of tumor, the growth rate and the weight of tumor, and the expression of SRPK2 and

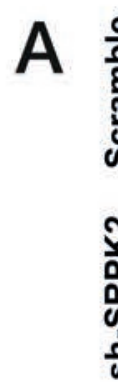

B
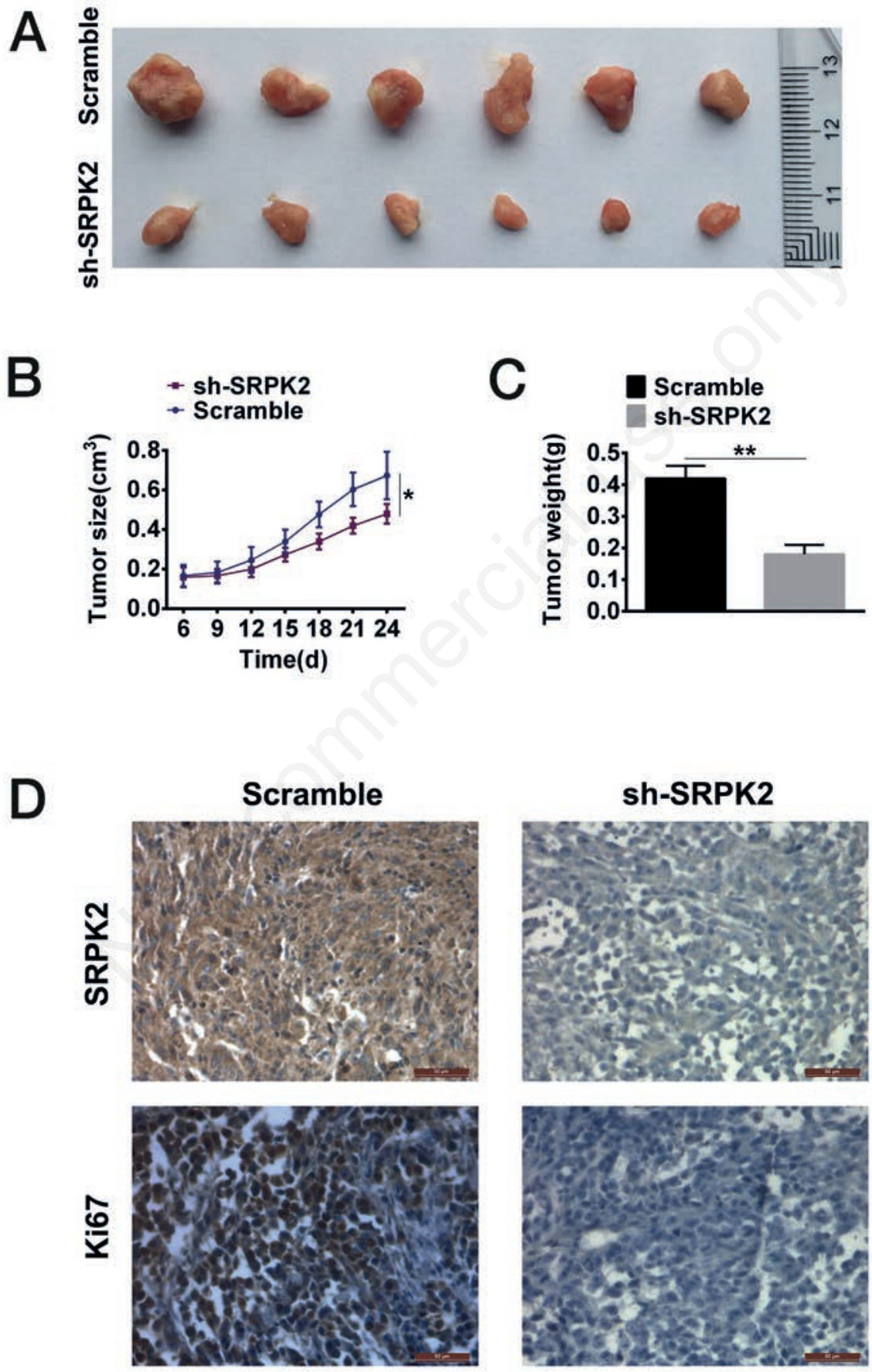

Figure 7. Knockdown of SRPK2 inhibits tumor growth in vivo. A-C) Loss of SRPK2 also remarkably decreased the weight of xenografted tumors and volume growth rates in vivo, compared with Scramble. D) IHC analysis in the xenografted tumors showed the notable reduction of total SRPK2 in vivo, Ki-67 immunohistochemistry for the xenografted tissues confirmed the suppressed proliferation of A549 cells with SRPK2 silence; nuclei stained brown indicated positive detection of Ki-67. 
Ki-67 are all decreased. These results demonstrated that the expression of SRPK2 affects tumor growth in vivo and also demonstrates the ability of SRPK2 to drive tumor progression.

In conclusion, the current study suggests that SRPK2 is upregulated in NSCLC and the higher SRPK2 expression was linked with survival rate of NSCLC patients. Functional experiments showed that SRPK2 might play a crucial role in NSCLC tumor progression. SRPK2 phosphorylates SC35, and phosphorylated SC355 activates E2F1, which regulates the transcriptional function of cycle-associated proteins. These findings of the present study also fully elaborate the precise outcomes of SRPK2 in NSCLC. Our data demonstrate that SRPK2 may be used as a prognostic indicator and therapeutic target for NSCLC.

\section{References}

1. Torre LA, Bray F, Siegel RL, Ferlay J, Lortet-Tieulent J, Jemal A. Global cancer statistics, 2012. CA Cancer J Clin 2015;65:87-108.

2. Millett RL, Elkon JM, Tabbara IA. Directed therapies in anaplastic lymphoma kinase-rearranged non-small cell lung cancer. Anticancer Res 2018; 38:4969-75.

3. Siegel RL, Miller KD, Jemal A. Cancer statistics, 2017. CA Cancer J Clin 2017;67:7-30.

4. Torre LA, Siegel RL, Ward EM, Jemal A. Global Cancer incidence and mortality rates and trends - An update. Cancer Epidemiol Biomarkers Prev 2016;25: 16-27.

5. Herbst RS, Heymach JV, Lippman SM. Lung cancer. N Engl J Med 2008;359: 1367-80.

6. Siegel R, Naishadham D, Jemal A. Cancer statistics, 2013. CA Cancer J Clin 2013;63:11-30.

7. Mavrou A, Brakspear K, HamdollahZadeh M, Damodaran G, Babaei-Jadidi $\mathrm{R}$, Oxley J, et al. Serine-arginine protein kinase 1 (SRPK1) inhibition as a potential novel targeted therapeutic strategy in prostate cancer. Oncogene 2015;34: 4311-9.

8. Graveley BR. Sorting out the complexity of SR protein functions. RNA 2000;6:1197-211.

9. Zhou Z, Qiu J, Liu W, Zhou Y, Plocinik RM, Li H, et al. The Akt-SRPK-SR axis constitutes a major pathway in trans- ducing EGF Signaling to regulate alternative splicing in the nucleus. Mol Cell 2018;71:872.

10. Ngo JC, Chakrabarti S, Ding JH, Velazquez-Dones A, Nolen B, Aubol BE, et al. Interplay between SRPK and $\mathrm{Clk} /$ Sty kinases in phosphorylation of the splicing factor ASF/SF2 is regulated by a docking motif in ASF/SF2. Mol Cell 2005;20:77-89.

11. Kuroyanagi N, Onogi H, Wakabayashi T, Hagiwara M. Novel SR-protein-specific kinase, SRPK2, disassembles nuclear speckles. Biochem Biophys Res Commun 1998;242:357-64.

12. Jang SW, Yang SJ, Ehlen A, Dong S, Khoury H, Chen J, et al. Serine/arginine protein-specific kinase 2 promotes leukemia cell proliferation by phosphorylating acinus and regulating cyclin A1. Cancer Res 2008;68:4559-70.

13. Jang SW, Liu X, Fu H, Rees H, Yepes M, Levey A, et al. Interaction of Akt-phosphorylated SRPK2 with 14-3-3 mediates cell cycle and cell death in neurons. J Biol Chem 2009;284:24512-25.

14. Wang J, Wu HF, Shen W, Xu DY, Ruan TY, Tao GQ, et al. SRPK2 promotes the growth and migration of the colon cancer cells. Gene 2016;586:41-7.

15. Zhuo YJ, Liu ZZ, Wan S, Cai ZD, Xie JJ, Cai ZD, et al. Enhanced expression of SRPK2 contributes to aggressive progression and metastasis in prostate cancer. Biomed Pharmacother 2018;102:531-8.

16. Gout S, Brambilla E, Boudria A, Drissi R, Lantuejoul S, Gazzeri S, et al. Abnormal expression of the pre-mRNA splicing regulators SRSF1, SRSF2, SRPK1 and SRPK2 in non small cell lung carcinoma. PLoS One 2012; 7:e46539.

17. Liu H, Hu X, Zhu Y, Jiang G, Chen S. Up-regulation of SRPK1 in non-small cell lung cancer promotes the growth and migration of cancer cells. Tumor Biol 2016;37:7287-93.

18. Dimova DK, Dyson NJ. The E2F transcriptional network: old acquaintances with new faces. Oncogene 2005;24: 2810-26.

19. Harbour JW, Dean DC. The Rb/E2F pathway: expanding roles and emerging paradigms. Genes Dev 2000;14:2393409.

20. Eymin B, Gazzeri S, Brambilla C, Brambilla E. Distinct pattern of E2F1 expression in human lung tumours: E2F1 is upregulated in small cell lung carcinoma. Oncogene 2001;20:1678-87.
21. Salon C, Merdzhanova G, Brambilla C, Brambilla E, Gazzeri S, Eymin B. E2F1, Skp2 and cyclin E oncoproteins are upregulated and directly correlated in high-grade neuroendocrine lung tumors. Oncogene 2007;26:6927-36.

22. Merdzhanova G, Edmond V, De Seranno S, Van den Broeck A, Corcos L, Brambilla C, et al. E2F1 controls alternative splicing pattern of genes involved in apoptosis through upregulation of the splicing factor SC35. Cell Death Differ 2008;15:1815-23.

23. Edmond V, Merdzhanova G, Gout S, Brambilla E, Gazzeri S, Eymin B. A new function of the splicing factor SRSF2 in the control of E2F1-mediated cell cycle progression in neuroendocrine lung tumors. Cell Cycle 2013; 12:1267-78.

24. Sasaki H, Endo K, Okuda K, Kawano O, Kitahara N, Tanaka H, et al. Epidermal growth factor receptor gene amplification and gefitinib sensitivity in patients with recurrent lung cancer. J Cancer Res Clin Oncol 2008;134569-77.

25. McMillen E, Ye F, Li G, Wu Y, Yin G, Liu W. Epidermal growth factor receptor (EGFR) mutation and p-EGFR expression in resected non-small cell lung cancer. Exp Lung Res 2010;36: 531-7.

26. Kim DS, Park KM, Won YS, Kim JY, Lee JK, Kim JG, et al. Occurrence and prognosis of symptomatic venous thromboembolism in colorectal cancer surgery patients. Vasc Specialist Int 2014;30:49-55.

27. Loh BJ, Cullen CF, Vogt N, Ohkura H. The conserved kinase SRPK regulates karyosome formation and spindle microtubule assembly in Drosophila oocytes. J Cell Sci 2012;125:4457-62.

28. Zheng Y, Fu XD, Ou JH. Suppression of hepatitis B virus replication by SRPK1 and SRPK2 via a pathway independent of the phosphorylation of the viral core protein. Virology 2005;342:150-8.

29. Muller H, Bracken AP, Vernell R, Moroni MC, Christians F, Grassilli E, et al. E2Fs regulate the expression of genes involved in differentiation, development, proliferation, and apoptosis. Genes Dev 2001;15:267-85.

30. Johnson DG, Degregori J. Putting the oncogenic and tumor suppressive activities of E2F into context. Curr Mol Med 2006;6:731-8. 